\title{
Contact thermography method and TOT and DOT apparatus as an alternative to conventional devices and techniques used in diagnosing of breast cancer
}

Keywords: tomography volumetric thermal, volumetric thermal diagraph, computer tomography; mammary gland, magnetic resonance tomography, benign tumor; breast cancer, fibro cystic breast disease

Abbreviations: TOT, tomography volumetric thermal; DOT, volumetric thermal diagraph; $\mathrm{CM}$, conventional diagnostic methods adopted in clinics at the present time; CT, computer tomography; MG, mammary gland; MRI, magnetic - resonance tomography; RM, X-ray mammography; DT, distant thermal imaging; PA, area of pathology; CRT, cancerous tumor; BT, benign tumor; $\mathrm{BC}$, breast cancer; $\mathrm{FBD}$, fibro cystic breast disease

\section{Introduction}

Modern medicine actually offers a person an alternative - to maintain health through a preventive regular examination, or in case of a disease consult a doctor and then, on his recommendation, conduct adequate treatment. However, if this disease turns out to be cancerous, the situation can be quite threatening for the patient, since the manifestation of such a disease occurs with the development of the tumor to stages, when the life-time of the patient is already shortened. For example, the five-year survival rate of patients with stage IV breast cancer does not exceed $10 \%$ of the number of cases, and if breast cancer is detected in stage I, 90 to $95 \%$ of patients experience a 5 -year period after surgery. ${ }^{1}$ The time from the appearance of the preclinical stage to the stage when the tumor disease manifests about three-fourths of the total time of the disease cycle before the lethal stage. ${ }^{2}$ The duration of the cycle itself is determined by the degree of malignancy of the tumor, estimated by the time of doubling cancer cells.

Modern medicine recommends mammography examinations for breast cancer once or twice a biennium, arguing that the mammary gland undergoes the involution stage by the age of 40 when radiation mammography becomes relatively effective. ${ }^{3}$ However, breast cancer, as practice shows, can also be manifested with pubertal age, ${ }^{4}$ when the tumor is diagnosed with biopsy and results in a mastectomy according to its results. The largest mammologist in Russia, professor L.D. Lindenbraten, assessing the current situation with breast cancer in 1979, spoke about this: "Timely detection of breast cancer ensures the preservation of life, the use of organ-saving treatment methods and reducing the costs of diagnostics and therapy. National and regional programs for mass screening of the female population and the creation of federal and regional registers of people screened and breast cancer patients are needed" [4, page 7]. These wishes are have actuality even now, despite the emergence of alternative techniques and equipment described below.

The disadvantages of the conventional techniques and equipment

The disadvantages of the most widely used in the diagnosis of breast
Volume 3 Issue 3 - 2017

Klukin L

LLC "Modern computer medical equipment" Ltd, Russia

Correspondence: Klukin L, LLC "Modern computer medical equipment" Ltd, Moscow, Russia, Email klukin32@mail.ru

Received: October 02, 2017 | Published: October 20, 2017

cancer are due to various reasons. These methods (X-ray, ultrasound) provoke mutations in different degrees by exposure to tissue cells and this can lead to the appearance of tumor neoplasms, ${ }^{5,6}$ his circumstance prevents the possibility of their use in tumor screening, and, in turn, does not provide an opportunity to assess the degree of malignancy of breast cancer and, therefore, to monitor its development operatively. In the worst case, this leads to a loss of time and the tumor develops further. And, if the doubling of cancer cells occurs in a short time, the transition to stage III or IV will take several months. ${ }^{7}$ Methods of remote thermography using a thermal imager are safe, but not informative, because of the high error in estimating the temperature gradients on the patient's breast skin, which makes it impossible to detect small tumors or located in the depth of the body in the area of their projection onto the skin. ${ }^{8}$ These methods and equipment, including X-ray and magnetic resonance tomography, can work only when there is a tumor whose structure and its content is different from the surrounding tissues. This means their inability to determine the coordinates of the preclinical stage before of the occurrence of breast cancer. All these and other drawbacks of conventional methods are well known from the current manuals on mammology ${ }^{3}$ and from the practice of their application. ${ }^{9}$

\section{About technique of application of TOT and DOT in early diagnostics of a cancer of a mammary gland}

Using of these apparatus is based on a new principle in the survey, which consists in using the directionality of the heat flux in the human body orthogonally to the skin surface. At the same time, if pathologies are present in the path of this flow in the form of tumor neoplasms, then in their projection the temperature gradients appear on the patient's skin, the sign and magnitude of which are uniquely related to the three-dimensional coordinates and the temperature inside the tumorous neoplasm..$^{10,11}$ Figure 1, respectively, shows the heads of the device TOT (prototype) and DOT1-1 (the last modified sample), with which you can conduct an examination in an offline mode. Figure 2, respectively, shows a technique for examining the mammary glands in a "lying" posture (universal for any bust size) and in a "sitting" position for the size of the bust from the first to the second. There are elastic masks that fix holes in the skin, where temperature gradients are measured. 


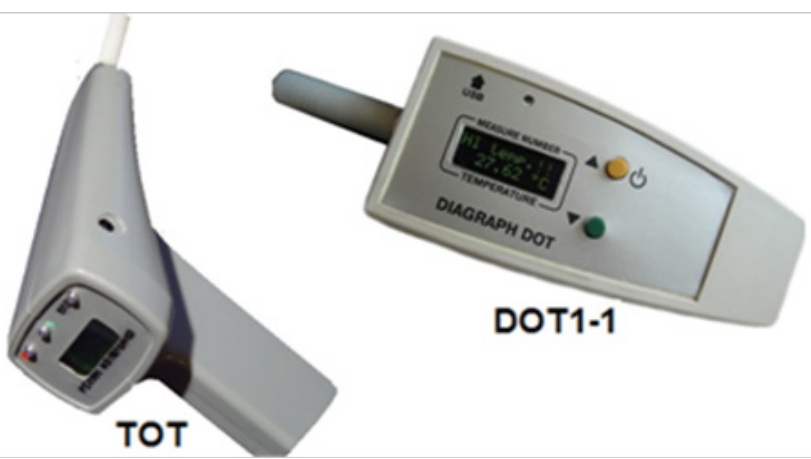

Figure I Appearance of the autonomous heads of TOT and DOT.

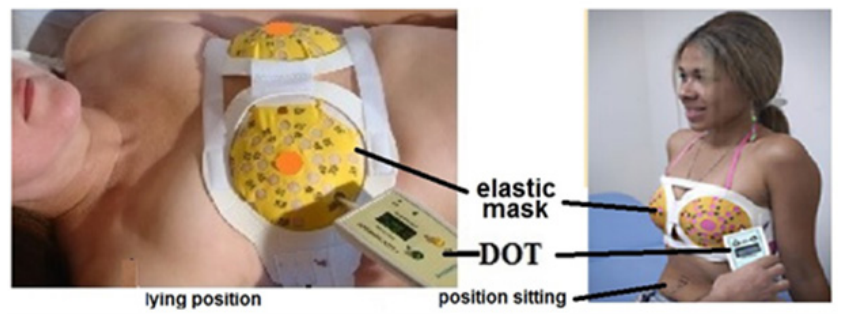

Figure 2 Inspecting the patients in the lying position and in sitting with the DOT

\section{About using of TOT and DOT}

As a result of the survey, the monitor displays information in the form of a two-dimensional mammogram (Figure 3), where a cancer is detected in the left breast, or a mammogram in the $3 \mathrm{D}$ space of the same mammary gland (Figure 3 Position 3), with a prominent tumor in this volume. In this case, examples of automatic conclusion about the "norm" or benign tumor - fibrocystic disease (FBD), dangerous as a precursor of the $\mathrm{BC}$, or the presence of breast cancer in the mammary glands, can be shown. With regard to prior experience of the application of the apparatuses and THE DOT to assess the risk of breast cancer, were obtained of the standard form of the tumor in malignant forms shown in Figure 4. In the early years of using DOT in mammology, the diagnosis of the disease was based on the use of standard forms of breast cancer (Figure 4), but after the discovery of the effect of cyclic blood filling of the MG with a relatively short period, it was found that changes in the temperature field accompanying this cycle on the cutaneous surface of the breast can mask these typical forms. ${ }^{12}$ To avoid this, a table of data on the coordinates of tumor tumors in the breast, the temperatures at their centers, and the size of these areas was drawn on the monitor (Figure 3 Position 3). The temperature at their centers, and the size of these areas was drawn on the monitor (Figure 5). As the example confirming efficiency of modified DOT-diagnostics on Figure 5 is shown a finding of BC at patient M.,56 years, using this temperature signs and threedimensional co-ordinates. Mastektomy which has been done later, has confirmed this finding (see the top and bottom external of thermogram quadrants). The research in patients with suspected at breast cancer have shown that both criteria (the availability of model forms for breast cancer and an increased temperature gradient in the tumor) are stable factors, in connection with which the patient is at higher risk for breast cancer and to identify the potential course of the disease is required to conduct appropriate monitoring

In recent years this circumstance we believed sufficient medical basis for ascertaining the type of disease and taking appropriate measures to further treatment or monitoring of this disease. However, the current practice of using the technique of DOT showed that the symptom of prominence in neoplastic growths on the thermograms (Figure 1) optional for writing a medical case history of the patient has a malignant tumor. Moreover, in some clinical studies it was noted that the DOT method can be used in cases of excess of the temperature gradient in the DOT points of thermograms corresponding to the marked on it the poles of the isotherms ${ }^{6}$ regardless of these types of forms on the thermogram. ${ }^{12}$ All the examined patients, depending on the condition of the MG ("norm", FBD, BC) were divided into 5 groups, each of which consisted of 12 patients: group 1 "norm", group 2 with FBD in left MG, group 3 with FBD in right MG; group 4 with $\mathrm{BC}$ in the left $\mathrm{MG}$, and group 5 with $\mathrm{BC}$ in the $\mathrm{MG}$ right breast. (Nosology in the known that this form is often the precursor of $\mathrm{BC}^{2}$ ). For each patient from the groups, according to the data in Table 3 (in Figure 8), the graphs shown in Figure 6 were compiled. Further processing of these graphs was carried out by a program providing automatic issuance of a diagnosis of MG disease to the monitor in accordance with each of these groups (Figures $7 \&$ Figures 8 ). When form of FBD was included by us in the object of investigation. ${ }^{13}$ Useful properties of DOT in terms of monitoring and its high sensitivity can be illustrated by the example of early diagnosis of CS in patient K, 56 years old, who had applied for pain in the left breast.

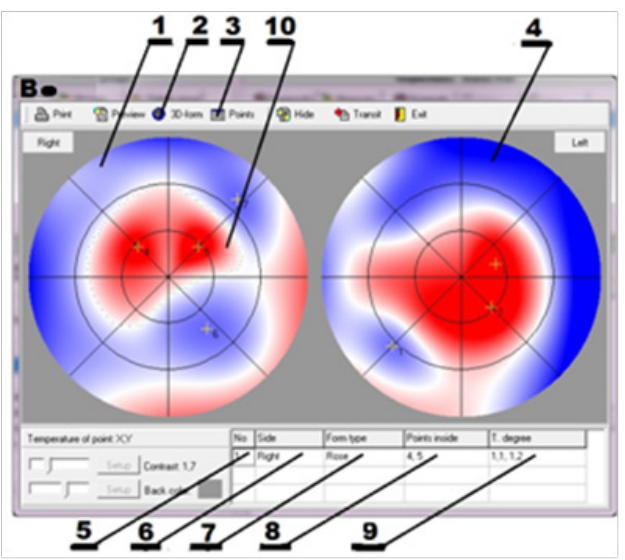

Figure 3 The mammogram of the patient: I-right breast, 2 - button switch to 3D, 3-table of data, 4-left breast, 5- zones PA, 6 - the breast, 7-type of PA, 8 numbers of PA zones, 9 - max gradients of temperature in PA zones, 10 -PA in form of "rose".

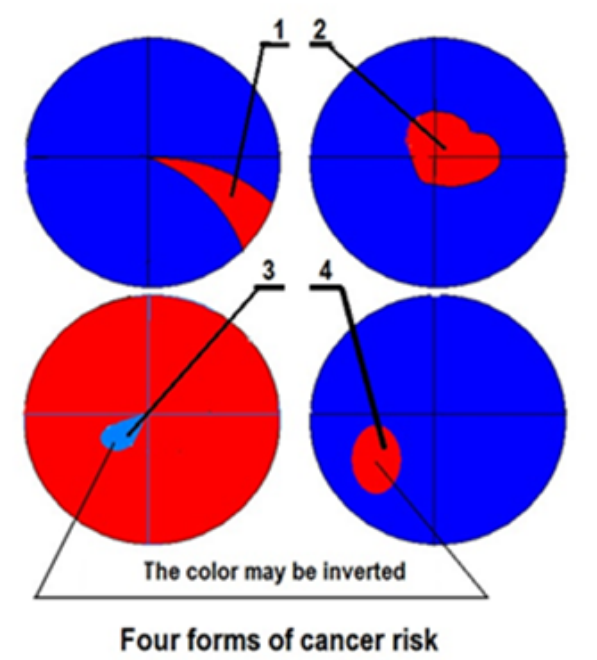

Figure 4 Forms of cancer risk: I- the wedge, 2-the rose. 3- the hammer, 4- the ellipse. 


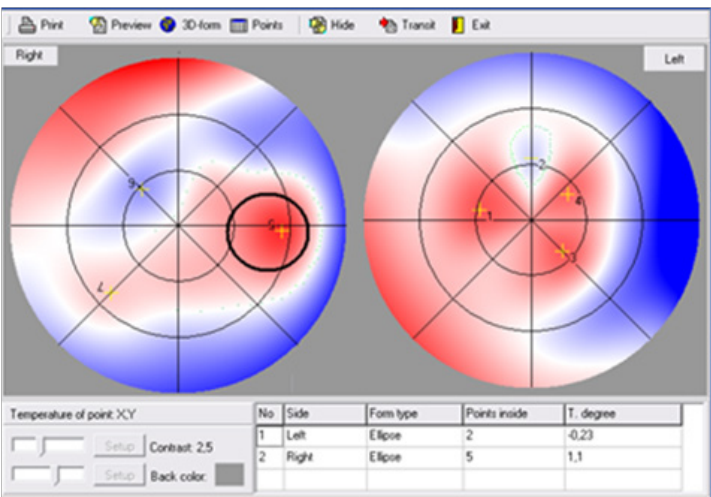

Figure 5 Allocated $B C$ on right MG: I - the right MG, 2 - the verified area of a cancer, 3 - left MG.

From Figure 9 we can see at A series plane thermograms where at the left MG at the border of the inner upper and lower quadrants there is a tumor of the "wedge" type with a size of about $3 \mathrm{~mm}$ with an excess temperature of $0.53^{\circ} \mathrm{C}(1)$. Checking this tumor about the possibility of having breast cancer, one should consider this mammogram in 3D space after the cursor has pressed the PA zone in a two-dimensional mammogram, we get a mammogram in 3D space (2). In accordance with the Instruction on DOT, patient $\mathrm{K}$. was sent to the mammalogist for the establishment of a biopsy of the presence of breast cancer by the criteria of temperature in the tumor and the shape of the "wedge". Since DOT provides a possible monitoring of the disease, the doctor prescribed the patient the intake of sodium selenite in doses providing prooxidant activity, ${ }^{14}$ apparently not without reason, considering the preclinical stage of the tumor. After the end of the next cycle, the patient $\mathrm{K}$. conducted a second examination, a two-dimensional mammogram of which is shown in Figure 9 (3). From Figure 9 in 3D it is evident that the tumor dislocation in the form of "wedge" was preserved on the same left breast and in the same coordinates, but became it is smaller $(2 \mathrm{~mm})$, and its temperature dropped to $0.37^{\circ} \mathrm{C}$. Subsequent tumor testing for breast cancer with the criteria specified above, showed the absence of breast cancer by the criteria. Since in medical technology any development introduced into medical practice is accompanied by the development of a document in the form of "Medical technology". It was compiled by LLC "Modern Computer Medical Equipment" based on the practical application of DOT in Russia, the EU, Armenia and Israel on 19 pages and coordinated with the Ministry of Health of Russia in 2007. According to this document, the main parameters characterizing DOT are:

i. Sensitivity of the method $-97.5 \%$.

ii. Specificity $87.1 \%$.

iii. Accuracy up to $88 \%]$.

iv. Contraindications to use - skin diseases.

\begin{tabular}{|c|c|c|c|c|c|c|c|}
\hline 1 & Let & 700 & $225 d e 0$ & 0.21 & $-1,1 d 00$ & $0.54 \mathrm{deg}$ & $6.65[72.44)$ \\
\hline 2 & Leth & $31 \%$ & $-45 d e g$ & 0.4 & $3,3 \mathrm{dog}$ & $1.7 \mathrm{deg}$ & $200:(128 \times 92)$ \\
\hline 3 & Lett & 25: & $21 \mathrm{deg}$ & 0.56 & $28 \mathrm{deg}$ & 1,4 deg & $128[132654]$ \\
\hline 4 & Ringth & $31 \%$ & $135 \mathrm{deg}$ & 0.27 & $2300 \mathrm{~g}$ & $1,1 \mathrm{deg}$ & $128(82858)$ \\
\hline 5 & Ringte & $31 \%$ & 45 deg & 0.25 & $2,4 \mathrm{deg}$ & $1.2 \mathrm{deg}$ & $9,4 \div[104.52]$ \\
\hline 6 & Ringte & $46 \%$ & $53 \mathrm{deg}$ & 0,49 & $-1,3 \mathrm{deg}$ & $0,65 \mathrm{deg}$ & $14 \div(34 \times 72)$ \\
\hline 7 & Ringte & $74 \%$ & $48 \mathrm{deg}$ & 0.3 & $-1.5 \mathrm{deg}$ & $0,74 \mathrm{deg}$ & $6.77(64 \times 48)$ \\
\hline
\end{tabular}

Figure 6 Table of coordinates and parameters of software in both MG. (The rows in this table reflect the contents of lines pos.3 shown in Figure 3).

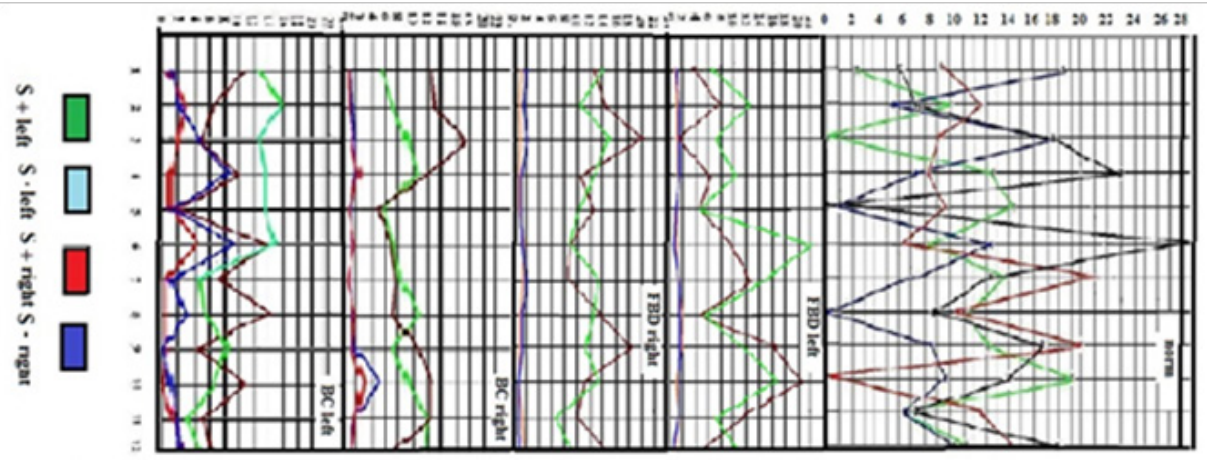

Figure 7 Data graphs for groups I-5.

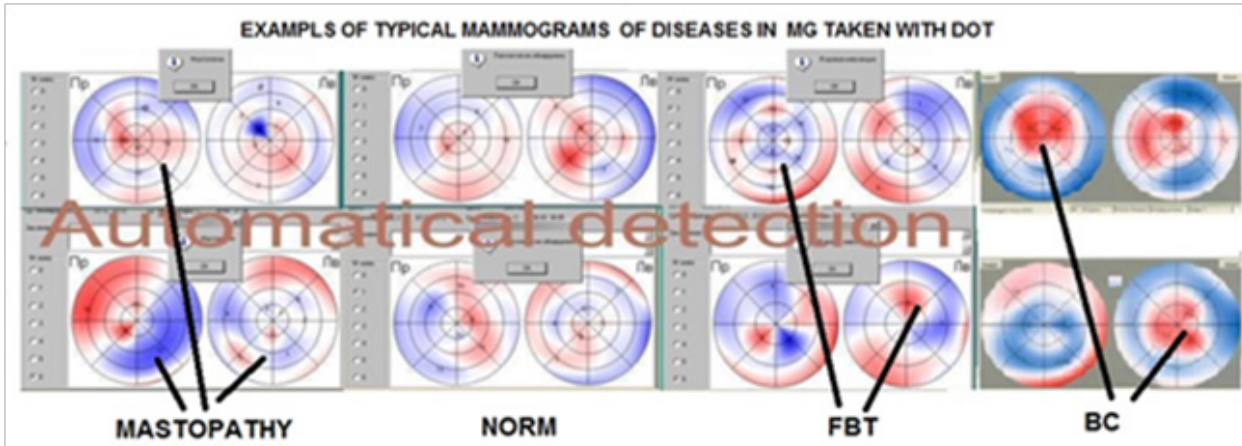

Figure 8 Examples of automatic issuance of a clinical conclusion about MG diseases on the computer screen. 

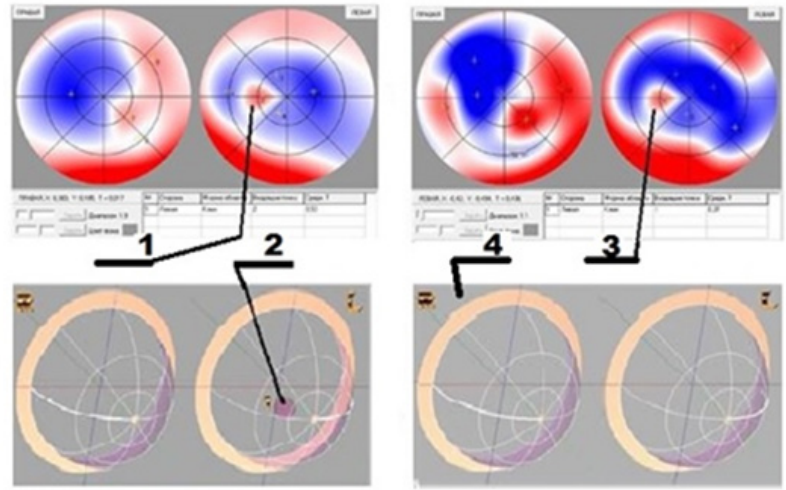

Figure 9 Display of survey data: A - two-dimensional mammogram; B-tumor in the 3D space of the left breast (see pos.B).

\section{Conclusion}

\section{The main advantages of the DOT method}

Its most important advantage is that it not only completely avoids the shortcomings of conventional methods, but also provides new opportunities as a result of careful testing in Russia and abroad, where data from medical centers in Russia ${ }^{12}$ and Israel played the main role: "Center Chaim Sheba" (Ramat - Gan) and "Erev" (Jerusalem). ${ }^{9}$ DOT:

i. It is absolutely safe for examination, since it does not affect the patient by any agent at all,

ii. allows you to restore and display in the three-dimensional space of the mammary gland types of tumors of various nosology's,

iii. allows you to automatically conclude about the "norm", benign tumor of fibrocystic mastopathy - a potential predecessor of breast cancer,

iv. allows to carry out arbitrarily frequent screening in order to establish its malignant tumor and optimize its therapy,

v. much cheaper than existing analogues,

vi. allows to diagnose cancer at the preclinical stage. ${ }^{14}$

\section{The practical using of the DOT}

Approbation of the DOT methodology was carried out in clinical conditions over the past 10 years in Russia, Greece, Armenia, Israel, the European Union, Argentina and Mexico. Certificates of conformity of Russia, the European Union, and the European quality mark "C", Israel and Argentina, as well as the highest awards of the most prestigious international exhibitions of inventions were received at the DOT-method (Brussels 2008, Geneva, 2009). The possibility to perform breast cancer screening at the preclinical stage of breast cancer with the help of DOT was demonstrated on the basis of an additional check conducted by the Ministry of Health of Russia, where it was stated that DOT also provides diagnostics in the preclinical stage of breast cancer, and there were no possible complications in the form of false positive and false negative results. ${ }^{15-17}$

\section{Acknowledgements}

None.

\section{Conflict of interest}

The author declares no conflict of interest.

\section{References}

1. Semiglazov VF, Krzhyvitsky PI. Radiation diagnosis of minimal breast cancer. Oncology issues. 2001;47(1):99-102.

2. Anderson S, Nielsen M. Essential histological finding in the female breast at autopsy, Early Breast Cancer. In: S Zander et al. editors. Germany: Springer Verlag; 1985. p. 52-53.

3. Ternovoi SK, Abduraimov AB. Radiation mammology. Russia: IG GEOTAR-Media; 2007. 128 p.

4. Lindenbraten LD, Burdina LM, Pinhosevich EG. Mammography (educational atlas). Russia: VIDAR; 1997.

5. Fran Lowry. Radiation exposure from annual mammography increases breast cancer risk in young high-risk women. Proceedings of the radiological society of North America, USA: Springer; 2009.

6. Klukin LM. The technique of using contact volumetric tomography for early diagnosis, screening, identification and monitoring of diseases of the population. Science and technological developments. 2010;89(2):1430 .

7. Semiglazov VF, Moiseenko VM, Chermondikova MF. Kinetics of breast cancer growth. Problems of oncology. 1989;2:288-293.

8. Klukin LM. Screening and monitoring of tumor neoplasms using the method of diagnostic contact thermography. Medical equipment. 2010;3:7-12.

9. Shikhman SM, Citrinebaum BI, Klukin LM, et al. Contact volumetric thermography of mammary glands. Endoscopic surgery. 2007;6:39-44.

10. Klukin LM. Method and device for temperature diagnostics of bioobject pathologies. US Pat US 7,214,194 B2; 2016.

11. Klyukin LM. A new method of non-invasive early, absolutely safe differential diagnosis of diseases in the human body and their monitoring. Russia: United All-Russian Scientific Bulletin; 2016.

12. LM Klyukin. Influence of the spatial-dynamic processes of blood filling of female mammary glands on the preclinical diagnosis of cancer by the method of scanning contact thermography. STP. 2014;93(4):3-16.

13. Klyukin LM. DOT-Diagnostics in the differential evaluation of a number of nosologies in the human body. Scientific and Practical Journal. 2016;5:21-35.

14. Screening express diagnostics and monitoring of tumoral neoplasms in the mammary glands. Diagnosis and preclinical screening of breast cancer. Ministry of health of the Russian federation, Russia: Springer; 2010.

15. Klyukin LM. Method for semiautomatic diagnosis of mammary gland pathologies. Patent of the Russian Federation No. 26222362, Russia; 2017.

16. Sviridov SP, SH B Kashia, Obukhova OA, et al. Possibilities of essential selenium in oncology. NN Blokhin; 2012. 23 p.

17. Methodical recommendations on the DTP. The use of a $3 D$ thermal "DOT" diagram for screening express diagnostics and monitoring tumor tumors using an absolutely safe method of scanning a patient's body. Russia: Ministry of Health of the Russian Federation; 2009. 18 p. 